\title{
Signatures of Relativistic Helical Motion in the Rotation Measures of Active Galactic Nucleus Jets
}

\section{Citation}

Broderick, Avery E., and Abraham Loeb. 2009. "SIGNATURES OF RELATIVISTIC HELICAL MOTION IN THE ROTATION MEASURES OF ACTIVE GALACTIC NUCLEUS JETS." The Astrophysical Journal 703 (2): L104-8. https://doi.org/10.1088/0004-637x/703/2/L104.

\section{Permanent link}

http://nrs.harvard.edu/urn-3:HUL.InstRepos:41393143

\section{Terms of Use}

This article was downloaded from Harvard University's DASH repository, and is made available under the terms and conditions applicable to Other Posted Material, as set forth at http:// nrs.harvard.edu/urn-3:HUL.InstRepos:dash.current.terms-of-use\#LAA

\section{Share Your Story}

The Harvard community has made this article openly available.

Please share how this access benefits you. Submit a story.

Accessibility 


\title{
SIGNATURES OF RELATIVISTIC HELICAL MOTION IN THE ROTATION MEASURES OF ACTIVE GALACTIC NUCLEUS JETS
}

\author{
Avery E. Broderick ${ }^{1}$ and Abraham Loeb ${ }^{2}$ \\ ${ }^{1}$ Canadian Institute for Theoretical Astrophysics, 60 St. George Street, Toronto, ON M5S 3H8, Canada; aeb@ cita.utoronto.ca \\ 2 Institute for Theory and Computation, Harvard University, Center for Astrophysics, 60 Garden Street, Cambridge, MA 02138, USA \\ Received 2009 July 16; accepted 2009 August 24; published 2009 September 8
}

\begin{abstract}
Polarization has proven to be an invaluable tool for probing magnetic fields in relativistic jets. Maps of the intrinsic polarization vectors have provided the best evidence to date for uniform, toroidally dominated magnetic fields within jets. More recently, maps of the rotation measure (RM) in jets have for the first time probed the field geometry of the cool, moderately relativistic surrounding material. In most cases, clear signatures of the toroidal magnetic field are detected, corresponding to gradients in RM profiles transverse to the jet. However, in many objects, these profiles also display marked asymmetries that are difficult to explain in simple helical jet models. Furthermore, in some cases, the RM profiles are strongly frequency and/or time dependent. Here we show that these features may be naturally accounted for by including relativistic helical motion in the jet model. In particular, we are able to reproduce bent RM profiles observed in a variety of jets, frequencydependent RM profile morphologies, and even the time dependence of the RM profiles of knots in 3C 273. Finally, we predict that some sources may show reversals in their RM profiles at sufficiently high frequencies, depending upon the ratio of the components of jet sheath velocity transverse and parallel to the jet. Thus, multi-frequency RM maps promise a novel way in which to probe the velocity structure of relativistic outflows.
\end{abstract}

Key words: galaxies: jets - magnetic fields - polarization - radiative transfer - radio continuum: general techniques: polarimetric

\section{INTRODUCTION}

Polarization observations of relativistic jets have been instrumental in identifying the presence of large-scale ordered magnetic fields (Taylor \& Perley 1992; Taylor 1998, 2000; Lister et al. 1998; Zavala \& Taylor 2001, 2002, 2003, 2004; Asada et al. 2008a, 2008b; Kharb et al. 2009; O'Sullivan \& Gabuzda 2009). For nearly all active galactic nucleus (AGN) jets, Faraday rotation dominates the polarization signal, scrambling the polarization angles of the intrinsic emission on angular scales that are small compared to the jet width. This can be ameliorated by determining the rotation measure (RM), via multi-wavelength polarization observations, and then removing the associated distortion using the standard relation,

$$
\Psi_{\text {int }}=\Psi_{\text {obs }}-\mathrm{RM} \lambda^{2}
$$

where $\Psi_{\text {int,obs }}$ is the intrinsic/observed polarization angle. Typical RMs for AGN jets and cores range from $10 \mathrm{rad} \mathrm{m}^{-2}$ to $10^{3} \mathrm{rad} \mathrm{m}^{-2}$, though it can reach $10^{5} \mathrm{rad} \mathrm{m}^{-2}$ in some extreme cases. Thus, at $1 \mathrm{GHz}$ frequencies, the intervening Faraday rotation results in polarization angle rotations of roughly $6^{\circ}-$ $600^{\circ}$. Nevertheless, once the Faraday rotation is solved for, via multi-wavelength polarization observations, and removed, most jets show remarkably uniform polarization maps, generally aligned with the jet axis. Both the polarization fraction and the orientation have been used to argue that in the observer frame jets are overwhelmingly toroidally dominated (Lyutikov et al. 2005).

Observations of the RM itself provide information about the intervening magnetized plasma. There are multiple potential sources for the RMs including the Galactic interstellar medium, the intracluster medium near the AGN, and cooler, less relativistic material surrounding the jet itself. The Galactic RM itself can be on the order of $10^{2}-10^{3} \mathrm{rad} \mathrm{m}^{-2}$ and varies considerably on large scales, presumably as a consequence of the structure of the Galactic magnetic field (Ekers et al. 1969; Men et al. 2008; Noutsos et al. 2008). The magnetic field strength and geometry within the intracluster medium is poorly constrained, though few sources lie sufficiently near the centers of cooling core clusters for this to dominate (Carilli \& Taylor 2002). In contrast, Faraday rotation intrinsic to the jet environment itself is expected to produce large RMs.

The morphology of the Faraday rotating medium surrounding AGN jets is still poorly constrained. While the lack of Faraday depolarization implies that the medium must be in the foreground and itself produce negligible emissions, presently it is not possible to distinguish between nearby unrelated ionized gas clouds and rotation within the near side of a jet sheath, presumably containing an ordered helical magnetic field. Free-free absorption from pc-scale foreground clouds has been detected in Cen A and NGC 1275, implying that these clouds exist in at least some sources (Jones et al. 1996; Walker et al. 2000). In these one would expect roughly random RM gradients, associated with the random orientations of the cloud magnetic fields. However, in a handful of cases thus far ordered RM gradients transverse to the jet axis have been observed, providing tantalizing evidence for the presence of helically magnetized sheaths surrounding AGN jets (Asada et al. 2008a, 2008b; Kharb et al. 2009; O'Sullivan \& Gabuzda 2009). It is this latter possibility that we restrict ourselves here.

For static, axisymmetric jet sheaths, the transverse RM profiles are roughly linear and symmetric about the jet axis, up to a uniform offset depending upon the magnetic pitch angle. While for many sources this is sufficient (Asada et al. 2008b; Kharb et al. 2009; O'Sullivan \& Gabuzda 2009), in a number of cases the RM profiles display significant asymmetries (Asada et al. 2008b; O'Sullivan \& Gabuzda 2009). Furthermore, 
these asymmetries can be both time and frequency dependent. While relativistic motion along the jet can reduce the RM in the observer frame considerably, it is unable to produce these asymmetric profiles (López 2006). Here we consider the effects of relativistic helical motion. This is expected theoretically in both magnetohydrodynamic and force-free jet models, as well as motivated by jet simulations, though these typically cannot probe the necessary dynamic range to make direct comparisons (Vlahakis \& Königl 2004; De Villiers et al. 2005; McKinney 2006; Komissarov et al. 2007; Tchekhovskoy et al. 2008; McKinney \& Blandford 2009). Within the context of a simple helical jet model, it is possible to reproduce both, the observed asymmetries and the frequency and temporal behavior of jet RM profiles, without appealing to asymmetric structures or special viewing angles.

\section{FARADAY ROTATION IN RELATIVISTIC BULK FLOWS}

Faraday rotation is a result of the different phase velocities of the two, nearly circularly polarized, ${ }^{3}$ electromagnetic eigenmodes of magnetized plasmas. The subsequent accrual of a phase difference results in a local rotation of the polarization plane. Critical to this interpretation is the assumption that the plasma eigenmodes propagate nearly adiabatically. While changes in the underlying plasma properties (density and magnetic field) can lead to violations of this condition, for simplicity we will only consider cases in which the relevant plasma parameters change sufficiently slowly that these may be ignored (Enßlin 2003; A. E. Broderick \& R. D. Blandford 2009, in preparation). Furthermore, we will assume that the plasma smoothly joins the sub-relativistic material surrounding the jet, and thus will not consider the relativistic aberration of the resultant polarization. In this case, locally, the polarization angle, $\Psi$, changes by an amount identical to the phase difference accrued, $\Phi$. That is, in the plasma rest frame

$$
d \Psi=d \Phi=\frac{16 \pi^{3} e^{3}}{m_{e}^{2} c^{2} \tilde{v}^{2}} n \tilde{\mathbf{B}} \cdot d \tilde{\boldsymbol{\ell}}
$$

where $n$ and $\tilde{\mathbf{B}}$ are the proper electron density and rest-frame magnetic field, $\tilde{v}$ is the rest-frame frequency, and $d \tilde{\ell}$ is the rest-frame distance element. Since the total accrued phase is a Lorentz scalar, and we assume the plasma smoothly joins a non-relativistic flow, we obtain

$$
\Delta \Psi=\frac{16 \pi^{3} e^{3}}{m_{e}^{2} c^{2}} \int \frac{n}{\tilde{v}^{2}} \tilde{\mathbf{B}} \cdot d \tilde{\boldsymbol{\ell}} .
$$

Presently, this integral is performed in the comoving frame of the plasma. However, we may use the scalar nature of $\Psi$ to rewrite it in terms of the observed frequency, $v$, and an integral in the observers frame, $d \ell$. To do this, let us begin by defining the

\footnotetext{
3 This is generally true only for cold, ionic plasmas. In pair plasmas the eigenmodes are linear, while for plasmas with relativistic temperatures or substantial non-thermal components, the eigenmodes are typically significantly elliptical. However, the detection of RM gradients across AGN jets provides strong evidence for the presence of a significant, if not dominant, ionic component in the plasma surrounding the jet. For this reason, we have ignored the possible presence of a pair plasma in our analysis. Should the jet sheath be comprised of an admixture of the two, $n$ simply represents the excess electron density (Hall \& Shukla 2005). That the plasma is sufficiently cold is generally a good assumption far from internal shocks, and observationally supported by the lack of significant Faraday depolarization, and hence emission from within the Faraday rotating sheath.
}

plasma four-velocity, ${ }^{4} u^{\mu}=\gamma(1, \boldsymbol{\beta})$. In either the force-free or magnetohydrodynamic prescriptions, the electric field vanishes in the plasma frame, and thus $F^{\mu v} u_{v}=0$, where $F^{\mu v}$ is the electromagnetic field tensor. This implies that $F^{\mu \nu}=\epsilon^{\mu \nu \alpha \beta} u_{\alpha} b_{\beta} / 2$, where $b^{\mu}=\left(b^{t}, \mathbf{b}\right)$ is the four-vector corresponding to the magnetic field in the plasma rest frame and orthogonal to $u^{\mu}$. Due to the latter condition, the components of $b^{\mu}$ are not independent, and in particular $b^{t}=\boldsymbol{\beta} \cdot \mathbf{b}$. Finally, let us define the electromagnetic wavevector, $k^{\mu}=2 \pi v(1, \hat{\mathbf{k}})$, which is related to the electromagnetic wave trajectory, $x^{\mu}(\eta)$, by $d x^{\mu} / d \eta=k^{\mu}$. This implies that the line element as measured in the plasma frame is $d \tilde{\ell}^{\mu}=d x^{\mu}+u_{v} d x^{\nu} u^{\mu}=\left(k^{\mu}+u_{\nu} k^{\nu} u^{\mu}\right) d \eta$, and thus, $d \tilde{\ell}=$ $-u_{\mu} k^{\mu} d \eta$. Similarly, in the lab frame, $d \ell=k^{t} d \eta$. Therefore,

$$
\begin{gathered}
\tilde{v}=-\frac{u^{\mu} k_{\mu}}{2 \pi}=\gamma(1-\boldsymbol{\beta} \cdot \hat{\mathbf{k}}) v, \\
\tilde{\mathbf{B}} \cdot d \tilde{\boldsymbol{\ell}}=b_{\mu} d \tilde{\ell}^{\mu}=(\hat{\mathbf{k}}-\boldsymbol{\beta}) \cdot \mathbf{b} d \ell .
\end{gathered}
$$

Inserting these into the expression for rotation of the polarization gives

$$
\Delta \Psi=\frac{16 \pi^{3} e^{3}}{m_{e}^{2} c^{2}} \frac{1}{v^{2}} \int n \frac{(\hat{\mathbf{k}}-\boldsymbol{\beta}) \cdot \mathbf{b}}{\gamma^{2}(1-\boldsymbol{\beta} \cdot \hat{\mathbf{k}})^{2}} d \ell .
$$

The associated rotation measure is then simply

$$
\mathrm{RM} \equiv \frac{\partial \Delta \Psi}{\partial \lambda^{2}}=0.812 \int n \frac{(\hat{\mathbf{k}}-\boldsymbol{\beta}) \cdot \mathbf{b}}{\gamma^{2}(1-\boldsymbol{\beta} \cdot \hat{\mathbf{k}})^{2}} d \ell \operatorname{rad~m}^{-2}
$$

where $n, b$, and $\ell$ are measured in $\mathrm{cm}^{-3}, \mu \mathrm{G}$, and $\mathrm{pc}$, respectively.

When $\beta=0$, this rotation measure reduces to the standard expression, with $n$ and $\mathbf{b}$ now corresponding the plasma density and magnetic field strength in the observer frame. However, when $\beta \neq 0$, some care must be taken in interpreting these quantities, as both are defined in the plasma rest frame. In particular, the observer frame magnetic field, $\mathbf{B}$, is related to b by

$$
\mathbf{B}=\gamma(\mathbf{1}-\boldsymbol{\beta} \boldsymbol{\beta}) \cdot \mathbf{b} \quad \Leftrightarrow \quad \mathbf{b}=\frac{1}{\gamma}\left(\mathbf{1}+\gamma^{2} \boldsymbol{\beta} \boldsymbol{\beta}\right) \cdot \mathbf{B},
$$

which may be found by inspecting $F^{\mu \nu}$ directly. For large $\gamma$, B will appear dominated by the components transverse to the plasma motion.

The relativistic motion of the plasma has two important consequences. The first is to change the magnitude of the magnetic field along the line of sight due to relativistic aberration, producing the velocity-dependent term in the numerator of Equation (6). The second is simply to Doppler-shift the relevant plasma frequency, signified by the denominator in Equation (6), resulting in a correspondingly higher or lower RM as measured in the observer frame. This second effect can produce profound variations in the RM when $\boldsymbol{\beta}$ passes nearly parallel to the line of sight. The ratio of the RM of approaching and receding plasma flows is roughly $4 \gamma^{2}$, which for even moderate Lorentz factors can be considerable.

\section{ROTATION MEASURE PROFILES OF JETS}

Having an expression for the relativistic rotation measure in hand, we now must specify the properties of the Faraday rotating medium. For illustrative purposes we adopt an extraordinarily

\footnotetext{
4 Henceforth, we set $c=1$ unless otherwise noted.
} 


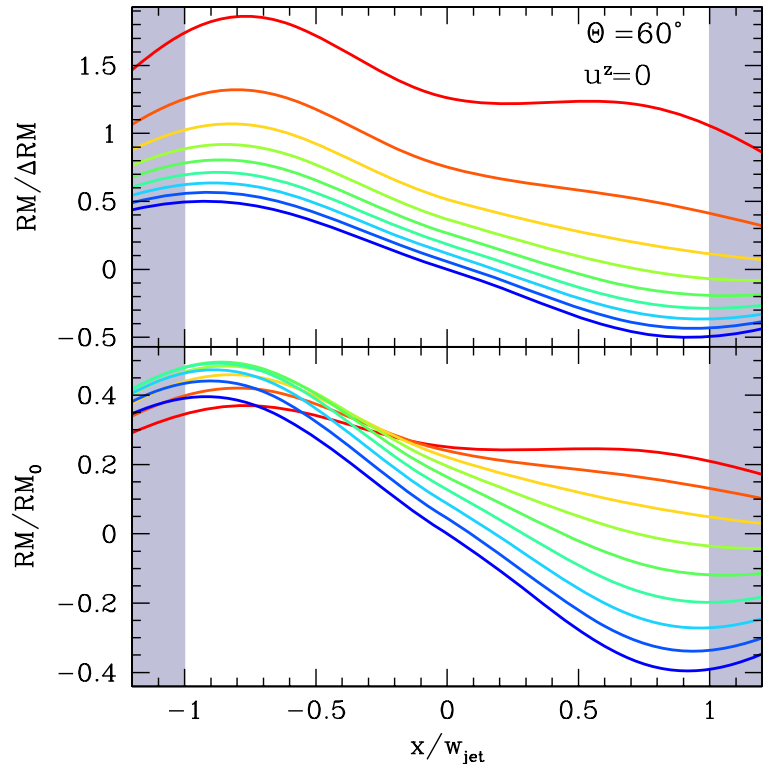

Figure 1. RM profiles of a static jet $\left(u^{z}, u^{\phi}=0\right)$ as viewed at $\Theta=60^{\circ}$, for magnetic pitch angle, $\alpha_{b}$, ranging from $10^{\circ}$ (red) to $90^{\circ}$ (blue) in steps of $10^{\circ}$. Top: the RM profile normalized such that the variation in the RM is unity. Bottom: the RM profiles in units of $81.2 \mathrm{rad} \mathrm{m}^{-2}$, corresponding to the RM generated by Faraday screen with electron density $1 \mathrm{~cm}^{-3}$, magnetic field $1 \mu \mathrm{G}$, and line-of-sight depth $1 \mathrm{pc}$. In both panels, the grayed regions show areas outside the jet core in projection, and thus presumably in which the RM cannot be measured.

simplified model: a cylindrically symmetric plasma sheath surrounding a jet core, appropriate at large distance from the jetlaunching region. While the cylindrical approximation is poor for viewing angles within the jet opening angle, for most cases of interest it is sufficient, allowing us to remove a degree of freedom from the modeling of the Faraday screen. The density peaks at some distance from the jet axis, falling exponentially beyond (our results are qualitatively independent of the radial density profile). We assume helical geometries for both the magnetic field and the plasma four-velocity. Explicitly, in cylindrical coordinates,

$$
\begin{gathered}
n=n_{0}\left(\frac{R}{w_{\mathrm{jet}}}\right)^{2} e^{-R^{2} / w_{\mathrm{jet}}^{2}} \\
b^{z}=b_{0} \cos \alpha_{b}, \quad b^{R}=0, \quad b^{\phi}=\frac{b_{0} \sin \alpha_{b}}{R}, \\
u^{z}=\beta \cos \alpha_{u}, \quad u^{R}=0, \quad u^{\phi}=\frac{\beta \sin \alpha_{u}}{R},
\end{gathered}
$$

where $R$ and $\phi$ are the cylindrical radius and polar angle, respectively, $w_{\text {jet }}$ is the characteristic width of the jet sheath, $n_{0}$ and $b_{0}$ are the density and field strength scaling (and are ultimately unimportant to the question of the RM asymmetry), $\beta$ is the velocity, and $\alpha_{u}$ and $\alpha_{b}$ are the velocity and magnetic field pitch angles, respectively. Finally, we define the angle between the line of sight and the jet axis to be $\Theta$, i.e., $\hat{k}^{z}=\cos \Theta$, $\hat{k}^{R}=\sin \Theta \cos \phi$, and $\hat{k}^{\phi}=-\sin \Theta \sin \phi / R$. Thus, $\Theta=0^{\circ}$ and $\Theta=90^{\circ}$ correspond to lines of sight along and orthogonal to the jet axis, respectively. For a variety of values for these parameters, we compute the RM as defined in Equation (6) as a function of transverse position, thereby constructing profiles across the jet.

The spatial variations in the density and orientation of the magnetic field necessarily lead to variation in the RM across the jet. In the limit of a static jet, where the plasma is at rest in the

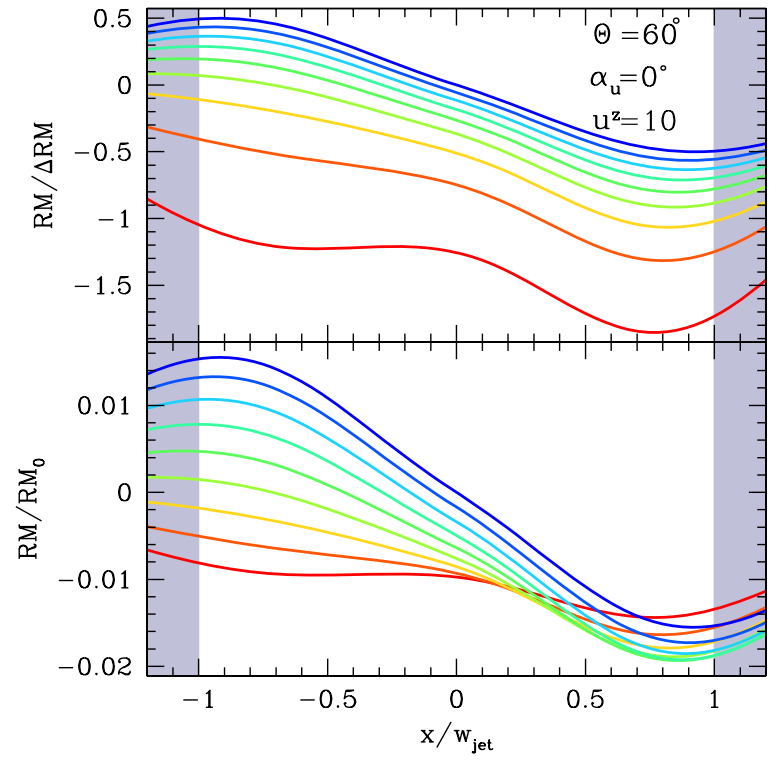

Figure 2. RM profiles of an ultra-relativistic, purely outflowing, jet, $u^{z}=10$ and $\alpha_{u}=0^{\circ}$, as viewed at $\Theta=60^{\circ}$, for magnetic pitch angle, $\alpha_{b}$, ranging from $10^{\circ}$ (red) to $90^{\circ}$ (blue) in steps of $10^{\circ}$. Top and bottom panels are defined as described in Figure 1.

observer frame, the RM profile is shown in Figure 1 for a variety of magnetic pitch angles, as viewed from $\Theta=60^{\circ}$. For purely toroidal magnetic fields $\left(\alpha_{b}=90^{\circ}\right.$, blue), the RM profile is symmetric and approximately linear, only departing from a line at the sheath boundaries due to the precipitous decrease in the electron density. In contrast, for magnetic fields nearly parallel to the jet $\left(\alpha_{b}=0^{\circ}\right.$, red $)$, the background density variation is clearly imprinted in the RM profile. Due solely to geometry, the degree to which this occurs depends upon $\Theta$, with the largest deviations at small $\Theta$ and $\alpha_{b}$. However, the absolute variation in the RM across the jet in this case is considerably reduced, and in the limit of $\alpha_{b}=0^{\circ}$ vanishes completely. More importantly, polarization maps of jets typically imply $R b^{\phi} / b^{z} \gtrsim 1$ (Lyutikov et al. 2005).

Jet sheaths with relativistic bulk motion along the jet axis, but without any helical motion, are similar to their static counterparts. In this case the primary consequence of the motion for the RM profiles, apart from the drastic reduction in the net $\mathrm{RM}$, is relativistic aberration, which effectively results in observers at oblique angles viewing the jet from behind. This is seen explicitly for an extreme case in Figure 2, in which the RM profiles are nearly identical to those for the static jet though rotated $180^{\circ}$. Most importantly, the sense of the RM gradient does not reverse as a consequence of the bulk motion. ${ }^{5}$ The RM profiles do not significantly differ from the case shown for $\gamma>3$, and for smaller velocities become even more degenerate in $\alpha_{b}$. Therefore, generally, within the context of axially symmetric jet models, magnetic fields with moderate pitch angles are incapable of producing strong asymmetric features in the RM profiles, though $\alpha_{b}$ and jet Lorentz factor do play a substantial role in determining the absolute RM.

In stark contrast, even moderately relativistic helical motion easily produces dramatic asymmetric features in RM profiles, independent of the magnetic field pitch angle, and in some cases reversing the sense of the RM gradient. As seen in Figure 3, this

\footnotetext{
5 For bulk motion along the jet to reverse the RM gradient would require $\Theta \gtrsim \gamma^{-1}$ while $\alpha_{b} \lesssim \gamma^{-1}$. While the former is almost certainly true, the latter would require magnetic field geometries that are nearly parallel to the jet axis.
} 

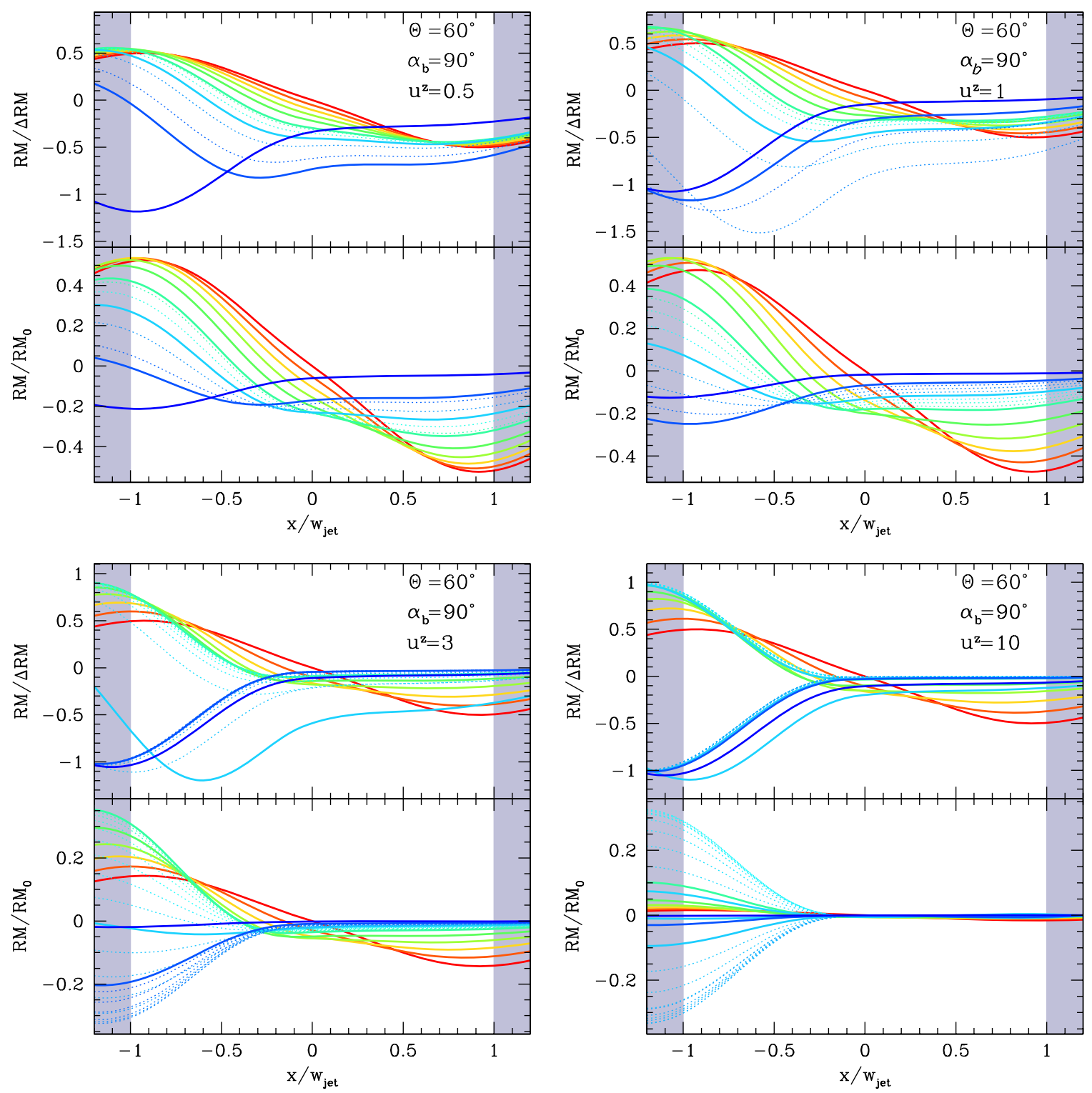

Figure 3. RM profiles of jets with helical velocity fields for $u^{z}=0.5$ (top left), $u^{z}=1$ (top right), $u^{z}=3$ (bottom left), and $u^{z}=10$ (bottom right). In all cases, $\alpha_{b}=90^{\circ}, \Theta=60^{\circ}$, and $\alpha_{u}$ ranges from $0^{\circ}$ (red) to $80^{\circ}$ (blue) in steps of $10^{\circ}$ for the solid lines. Dotted lines show the RM profiles for $\alpha_{u}$ distributed in steps of $0.1 / \gamma$ about $\Theta$, highlighting the transition due to relativistic aberration. Top and bottom panels are defined as described in Figure 1 . We choose to fix $u^{z}$, and not $\gamma$, since $u^{z}$ is directly measured for the jet core by observations of superluminal motion.

occurs both for trans-relativistic and ultra-relativistic jets, and for velocity pitch angles as low as $30^{\circ}$. Generic of relativistic helical motion is the noticeable bow in the RM profiles. This feature is only weakly dependent upon $u^{z}$ and present for $\alpha_{u} \gtrsim 30^{\circ}$ for $u^{z} \gtrsim 1$. Unlike similar features for non-rotating jets, this occurs for a wide range of absolute RMs.

When the revolving plasma in the jet sheath is approaching almost directly, i.e., $\boldsymbol{\beta}$ is within an angle $\gamma^{-1}$ of $\hat{\mathbf{k}}$, the direction in which relativistic aberration rotates the magnetic field rapidly changes (see the dotted lines in Figure 3). As a result, the RM evolves rapidly with $\alpha_{u}$. At the same time, the Doppler shift reaches its maximum, producing dramatic enhancements in the absolute RM, and for $\alpha_{u}$ very close to $\Theta$, complex RM profiles. However, for all but the slowest jets, this is restricted to such a small regime in viewing angle that it is relatively unlikely to be observed in practice. A much more important consequence is the reversal of the sign of the RM profile gradient at $\alpha_{u}=\Theta$. This is seen most clearly in the bottom two panels of Figure 3, in which the RM profiles associated with velocity fields with high pitch angles (blue) are nearly vertical reflections of those with low pitch angles (red). Thus, not only the symmetry of the RM profile is dependent upon $u^{\phi}$, but also the direction of the RM gradient itself.

\section{DISCUSSION}

The presence of relativistic helical motion in the Faraday rotating sheaths of AGN jets has a variety of specific and robust observational consequences, some of which have already been observed. These include the presence of significant, frequencydependent asymmetric features in the RM profiles, and even reversals in the RM gradient. These are illustrated for a simple jet sheath model in Figure 4. In this case, $u^{z}$ is constant along the jet while $u^{\phi}$, and thus $\alpha_{u}$, decreases with height. Low frequencies probe regions far from the AGN core (see, e.g., Blandford \& Konigl 1979), where the transverse velocities are low and RM profile is approximately linear. Conversely, 


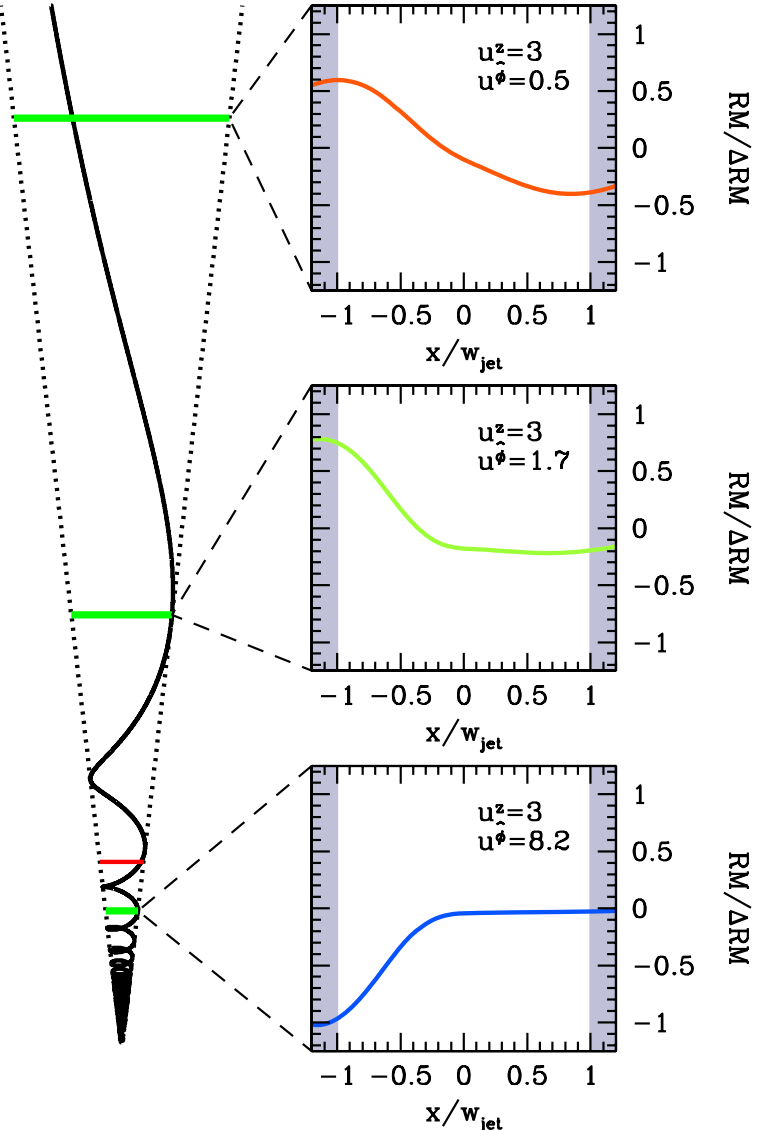

Figure 4. RM profiles of an evolving jet. The dotted lines show the envelope of the Faraday rotating sheath, while the helical solid line is the path taken by a particle as it propagates within the sheath. Throughout the jet $u^{z}=3$. Below the red bar, $\alpha_{u}>\Theta$ (again taken to be $60^{\circ}$ ), while above $\alpha_{u}<\Theta$. Positions along the jet where the RM profiles are shown at the right are marked by green bars. The RM profiles are colored as in Figure 3. Since the variation of the RM is dependent upon the magnetic field strength and plasma density in the jet, only the ratio $\mathrm{RM} / \Delta \mathrm{RM}$ is shown. In each plot of the profile, the components of the four-velocity parallel and transverse to the jet axis are listed.

intermediate frequencies probe regions closer to the AGN core, where the transverse velocities are higher, which for even modestly relativistic motions results in the characteristic bent RM profile. Sufficiently high frequencies may probe regions for which $\Theta<\alpha_{u}$, if they exist, and thus exhibit reversed RM profiles.

O'Sullivan \& Gabuzda (2009) present RM profiles for six blazars, as measured in a variety of frequency bands. In at least two cases, the RM profiles display the bent shape characteristic of helical motion: $1156+295$ and $1418+546$. In $1418+546$, this is seen clearly in an RM profile constructed from observations between $4.6 \mathrm{GHz}$ and $8.9 \mathrm{GHz}$. At higher frequencies, the RM profile shows evidence of being reversed, though the absolute $\mathrm{RM}$ is reduced by nearly 2 orders of magnitude, again consistent with the presence of relativistic motion. In contrast, at 4.68.9 GHz, 1156+295 exhibits a nearly linear RM profile, while at $7.9-15.4 \mathrm{GHz}$, the RM profile becomes dramatically bent. At higher frequencies $(12.9-43 \mathrm{GHz})$, it becomes roughly linear again, which may be evidence of reduced $u^{z}$ at small radii, a particularly fortuitous view of the transition to a reversed profile, or failure to fully resolve the RM gradient. In addition, $0954+658$ and $2200+420$ present dramatic changes in the absolute RM, including its overall sign, with changing observing frequency interval and radial distance from the AGN core. These are consistent with the observation of regions transitioning between ultra-relativistic and moderately relativistic helical motion close to the AGN core.

RM observations of knots in 3C 273 by Asada et al. (2008a) provide extraordinary examples of the bent profiles characteristic of helical motion (see Figure 4 of that paper). More interestingly, the RM profiles have evolved from 1995 to 2002, with the absolute RM increasing by roughly $40 \%$ and the location of the bend in the profile moving toward the jet center. This behavior is consistent with moderate slowing of the sheath during this period, i.e., a decrease in the sheath velocities of roughly $20 \%$ as they propagated outwards.

Unfortunately, most jet RM observations only marginally resolve the transverse jet structure. As a consequence, it may be premature to distinguish between Faraday rotation in nearby, though unrelated, gas clouds (see, e.g., Laing et al. 2008) and the ordered, helical sheath models discussed here. We note that $3 \mathrm{C}$ 273 is an exception, is clearly resolved, and provides a striking agreement with the profiles we present. However, space-based VLBI (VSOP-2; Hagiwara et al. 2009) and millimeter-VLBI (e.g., Broderick \& Loeb 2009; Doeleman et al. 2009) both promise order of magnitude improvements in resolution, and have the potential to resolve the nature of AGN jet Faraday screens conclusively.

We thank the anonymous referee for helpful comments. This work was supported in part by NSF grant AST-0907890.

\section{REFERENCES}

Asada, K., Inoue, M., Kameno, S., \& Nagai, H. 2008a, ApJ, 675, 79

Asada, K., Inoue, M., Nakamura, M., Kameno, S., \& Nagai, H. 2008b, ApJ, 682,798

Blandford, R. D., \& Konigl, A. 1979, ApJ, 232, 34

Broderick, A. E., \& Loeb, A. 2009, ApJ, 697, 1164

Carilli, C. L., \& Taylor, G. B. 2002, ARA\&A, 40, 319

De Villiers, J.-P., Hawley, J. F., Krolik, J. H., \& Hirose, S. 2005, ApJ, 620, 878

Doeleman, S. S., Fish, V. L., Broderick, A. E., Loeb, A., \& Rogers, A. E. E. 2009, ApJ, 695, 59

Ekers, R. D., Lequeux, J., Moffet, A. T., \& Seielstad, G. A. 1969, ApJ, 156, L21 Enßlin, T. A. 2003, A\&A, 401, 499

Hagiwara, Y., Fomalont, E., Tsuboi, M., \& Yasuhiro, M. (ed.) 2009, in ASP Conf. Ser. 402, Approaching Micro-Arcsecond Resolution with VSOP-2: Astrophysics and Technologies (San Francisco, CA: ASP)

Hall, J. O., \& Shukla, P. K. 2005, Phys. Plasmas, 12, 084507

Jones, D. L., et al. 1996, ApJ, 466, L63

Kharb, P., Gabuzda, D. C., O’Dea, C. P., Shastri, P., \& Baum, S. A. 2009, ApJ 694, 1485

Komissarov, S. S., Barkov, M. V., Vlahakis, N., \& Königl, A. 2007, MNRAS, 380,51

Laing, R. A., Bridle, A. H., Parma, P., \& Murgia, M. 2008, MNRAS, 391, 521

Lister, M. L., Marscher, A. P., \& Gear, W. K. 1998, ApJ, 504, 702

López, E. D. 2006, ApJ, 641, 710

Lyutikov, M., Pariev, V. I., \& Gabuzda, D. C. 2005, MNRAS, 360, 869

McKinney, J. C. 2006, MNRAS, 368, 1561

McKinney, J. C., \& Blandford, R. D. 2009, MNRAS, 394, L126

Men, H., Ferrière, K., \& Han, J. L. 2008, A\&A, 486, 819

Noutsos, A., Johnston, S., Kramer, M., \& Karastergiou, A. 2008, MNRAS, 386, 1881

O’Sullivan, S. P., \& Gabuzda, D. C. 2009, MNRAS, 393, 429

Taylor, G. B. 1998, ApJ, 506, 637

Taylor, G. B. 2000, ApJ, 533, 95

Taylor, G. B., \& Perley, R. A. 1992, A\&A, 262, 417

Tchekhovskoy, A., McKinney, J. C., \& Narayan, R. 2008, MNRAS, 388, 551

Vlahakis, N., \& Königl, A. 2004, ApJ, 605, 656

Walker, R. C., et al. 2000, ApJ, 530, 233

Zavala, R. T., \& Taylor, G. B. 2001, ApJ, 550, L147

Zavala, R. T., \& Taylor, G. B. 2002, ApJ, 566, L9

Zavala, R. T., \& Taylor, G. B. 2003, ApJ, 589, 126

Zavala, R. T., \& Taylor, G. B. 2004, ApJ, 612, 749 